\title{
Recycable complex catalysts immobilized on mercaptan-functionalized glass-polymer supports
}

\author{
Natalia Sienkiewicz $^{1}$ (D) Tomasz Szmechtyk ${ }^{1} \cdot$ Krzysztof Strzelec $^{1}$
}

Received: 20 February 2018/Revised: 3 April 2018/Accepted: 9 April 2018/

Published online: 19 April 2018

(C) The Author(s) 2018

\begin{abstract}
The use of the mercaptan-functionalized bisphenol A diglycidyl ether epoxy (base material) encapsulated on glass core as matrices for metal catalysts was investigated. Here, the acquiring knowledge of recyclable palladium catalysts for organic reaction which can be mechanically separated from the reaction media and next use as a heterogeneous catalyst in large-scale industries was the most important goal. Homogeneous $\mathrm{Pd}$ precursor $\left[\mathrm{PdCl}_{2}(\mathrm{PhCN})_{2}\right]$ has been heterogenized by attaching to prepared glass-polymeric supports via ligand exchange process. Collected research results indicate that mercaptans used to cure epoxy resin can greatly affect the catalytic properties of the epoxy resin with supported palladium catalyst. The epoxy system modified with mercaptans has a few possible catalytic coordination centers especially oxygen and sulfur to which the palladium ion can be bound. It should be noted that although various concentrations of palladium complex were used, the amount of metal attached to epoxide supports remained the same, that is below the sorption capacity of the polymers. Presented new type of glass-polymer supports comparing to other used corresponding organic carriers offers several practical advantages such as morphological and chemical structure of the matrix, which affect the properties of the heterogenized catalyst in the selected organic reactions: Heck reaction and hydrogenation reaction were investigated. The catalytic activity of all formulated systems was similar to homogeneous palladium precursor $\mathrm{PdCl}_{2}(\mathrm{PhCN})_{2}$. The resulting glass-polymeric matrices and heterogenized palladium catalysts were characterized by varied research techniques such as surface analysis technique (ToF-SIMS) and scanning electron microscopy with energy-dispersive X-ray analyzer (SEM-EDX). We used likewise the atomic absorption spectroscopy (AAS) method to quantify the amount of palladium loaded
\end{abstract}

Natalia Sienkiewicz

natalia.sienkiewicz@p.lodz.pl

1 Faculty of Chemistry, Institute of Polymer and Dye Technology, Lodz University of

Technology, Stefanowskiego 12/16, 90-924 Lodz, Poland 
in the recyclable support and also the BET method to determine specific pore size distribution parameters. Furthermore, XPS spectroscopy showed information about surface structure and chemical states in the palladium-supported catalysts.

Keywords Supported catalysts · Heterogeneous catalysis - Layered compounds · Microporous materials · Materials science $\cdot$ Polymers

\section{Introduction}

Encapsulation is a process to entrap active agents within a carrier material and it is a useful tool to improve delivery of molecules to give small capsules with many useful properties. The material inside the microcapsule is referred to as a core, internal phase, or fill, whereas the wall is sometimes called a shell, coating, or membrane. The technique of encapsulation depends on the type, physical and chemical properties of core and shell material. The chosen encapsulation technique should give a high encapsulation efficiency and loading capacity of actives, obtained capsules should also not exhibit aggregation or adherence and capsules should have a narrow particle size distribution without tails, threads or dents on the surface and the process should be suitable for industrial scale production.

Recently, there are many works which describe the encapsulation process, however, the list of their applications in catalysis has not been widely investigated. Several types of systems included supported salen-metal complexes which contain polyethylene ligands that characterized by advantage in increase in the rate of a chemical reaction also have extended stability toward acid. Matrix-bound $\mathrm{Cr}$ (III)$\mathrm{C}_{16} \mathrm{H}_{16} \mathrm{~N}_{2} \mathrm{O}_{2}$ and $\mathrm{Mn}(\mathrm{III})-\mathrm{C}_{16} \mathrm{H}_{16} \mathrm{~N}_{2} \mathrm{O}_{2}$ complexes accosted in a non-swelling solvent just like methanol did not bind with trifluoroacetic acid in methanol. The carrier characterization of presented earlier catalysts indicate that polyethylene ligands and a polyethylene matrices can have accessory advantage in recycling catalysts therein the solid state range of the recovered types may reduce uncommon reactions that disintegrate a prepared catalyst during its recycling [1].

Mennecke et al. used reduction reaction of Pd anions bound to an ion exchange resin inside microreactors to prepared palladium molecules. Degree of cross-linking and the density of ion exchange sites on the polymer/glass composites having influence and significantly affect the size and distribution of the Pd particles.

The catalytic characteristics of six further palladium-doped polymer/glass supports for hydrogenation reactions were explored alike under conventional conditions and microwave heating in the both likewise under constant flow conditions using the cyclohexene-which used as promoted transfer hydrogenation of ethyl cinnamate which was used as a model and chosen reaction. Therefore, towards the used heating method it was showed that catalysts that are composed of larger Pd particles present better under exposure to radiation conditions, whereas samples with smaller particle sizes which perform better under conventional heating conditions [2]. Recently, Pd particles have been immobilized on a Merrifield-type resin which is part of a Raschig ring-shaped glass/polymer composite material. This 
solid phase has successfully been employed under microwave $(\mu \mathrm{w})$ conditions in Suzuki-Miyaura reactions and other cross-coupling reactions. Additionally, Pd IIcomplex, which is insoluble in most solvents, can be incorporated inside an Ivory Kan teabag and repeatedly used in $\mu \mathrm{w}$-accelerated Suzuki-Miyaura cross-coupling reactions. The temperature had to be kept as low as $120^{\circ} \mathrm{C}$ to avoid decomposition of the polymeric material [3]. To receive the effortless, rapid, and clean removal of catalysts from reaction process, it is need to use the immobilization technique of metal nanoparticles in glass-polymer matrices. The catalyst supports contain a glass core are able to the mechanical removal from liquid reactions and an exterior superficies of support is suitable and can use for modification with mercaptanfunctionalized bisphenol A diglycidyl ether epoxy which allows to obtain carriers with specific properties. Jacinto prepared a magnetically amino-functionalized matrices with immobilized iridium species which are able to be regained or retrieved from liquid reactions and exhibited and characterized appropriate active hydrogenation reaction. Furthermore, a nonfunctionalized matrix and an analogous $\operatorname{Ir}^{0}$ catalyst have an upper degree of metal leaching in the liquid reaction products [4]. There have been reports of use of the functionalized epoxy resin encapsulated on magnetic core as matrices for Pd catalysts which can be removed from reaction by magnetic field. It was shown that functionalized epoxy resins can be used as coating for magnetic core in encapsulation process. On the basis of recycling efficiency tests, it was found that the all epoxy-supported palladium catalysts have a high catalytic stability for consecutive runs [5]. $\mathrm{Fe}_{3} \mathrm{O}_{4}$ nanoparticles were coated with diaminoglyoxime (DAG) as organic shell via a co-precipitation method. The $\mathrm{Fe}_{3} \mathrm{O}_{4} /$ DAG nanocomposites were effectively employed as support for immobilization of Pd nanoparticles. Herein, the immobilized Pd species on prepared support were really effective and efficient in the Suzuki reaction of varied aryl halides and particularly lower reactive aryl chlorides with phenyl boronic acid and addition characterized by the following advantages: (a) simple preparation; (b) easy separation after reaction by an external magnet; and (c) possibility of further use for several times without any meaningful reduction in its catalytic activity and stability [6]. The superparamagnetic $\mathrm{C}-\mathrm{Fe}_{3} \mathrm{O}_{4}-\mathrm{Pd}$ catalyst was successfully prepared by a one-pot solvothermal route, and it showed high catalytic activity in the aerobic oxidation of 5-hydroxymethylfurfural (HMF) into 2,5-furandicarboxylic acid (FDCA) in water under mild reaction conditions. Results demonstrated that reaction temperature and base concentration greatly affected not only the oxidation of HMF, but also its degradation. Under optimal reaction conditions, high HMF conversion of $98.2 \%$ and FDCA yield of $91.8 \%$ were obtained after $4 \mathrm{~h}$ at $80{ }^{\circ} \mathrm{C}$ with a $\mathrm{K}_{2} \mathrm{CO}_{3}$ / $\mathrm{HMF}$ molar ratio of 0.5 . Excellent results were also achieved by the oxidation of $\mathrm{HMF}$ over $\mathrm{C}-\mathrm{Fe}_{3} \mathrm{O}_{4}-\mathrm{Pd}$ catalyst in air and at room temperature with an appropriate reaction time. More importantly, the catalyst was easily recovered by an external magnet and reused without the loss of its catalytic activity [7]. Actually, epoxy systems have assorted and routinely applications as coating materials, adhesives and composites have dominant application position in contrast to other polymers. Epoxy resins used as matrices for complex catalyst are obtained by modification of epoxy networks by introduction of metal complexes into the structure of the cured support. So far there have been only a few statements on the use of epoxy resins as carriers of 
metal complex catalysts in organic synthesis for example epoxy resins doped with molybdenum, palladium, and ruthenium complexes have high catalytic stability in the processes of organic reactions (e.g., hydrogenation and hydroformylation) [8-10]. In our previous works, we proposed similar approach using bisphenol epoxy systems modified with ammonium ionic liquids as carrier for $\mathrm{PdCl}_{2}(\mathrm{PhCN})_{2} \mathrm{com}$ plex catalyst. The ionic liquids as hardeners for epoxy resins could greatly affect the activity of the catalyst without necessity of additional functionalization to improve ligand-binding affinity to metal complexes. Investigated catalysts showed good selectivity during prolonged use in the Heck reaction [11].

In the present study, we describe our approach to prepare the recyclable catalysts by immobilizing the palladium complex on the functionalized epoxy resin as coating for glass core. These catalysts were evaluated for their performance in the Heck and hydrogenation reaction. The direction of the research is consistent with the research conducted in the world [12-15].

\section{Experimental}

\section{Materials}

Epoxy resin bisphenol A epichlorohydrin medium molecular weight $<700$ Epidian 5 (EP5), epoxy equivalent 200, (viscosity 20000-30000 mPas at $23{ }^{\circ} \mathrm{C}$ ), triethylenetetramine (TETA), Ciech Sarzyna Poland. Mercaptans used to modification of epoxy resin (Sigma-Aldrich). Iodobenzene (Sigma-Aldrich), triethylamine (Ciech Sarzyna), methyl acrylate (Sigma-Aldrich) and $N$-methylhylpyrrolidone (ABCR GmbH) were used for Heck reaction. Cinnamaldehyde (Sigma-Aldrich) was used for hydrogenation reaction. All organic reagents were used as received without further purification.

\section{Preparation of the carriers}

The carrier was prepared using encapsulation technique of glass core by mercaptan functionalized epoxy resin (Fig. 1). The Fensky glass rings size of $2.5 \mathrm{~mm}$ were used as recyclable cores. In these studies, the multifunctional thiols: 3,6-dioxa-1,8octanedithiol (DODT), trimethylolpropane tris(3-mercaptopropionate) (TTMA), pentaerythritol tetrakis(3-mercaptopropionate) (PETMP) were applied to functionalization of epoxy resin. The chemical structure of the used hardeners DODT, TTMA and PETMP were presented in this paper in Fig. 2a-c.

$5 \mathrm{~g}$ of glass rings with $150 \mathrm{ml}$ of acetone were placed in a two-necked flask equipped with a mechanical stirrer. Then, the first portion of previously prepared mixture of an epoxy resin dissolved in a small amount of acetone, curing agent TETA and mercaptan (DODT, TTMA or PETMP) was added. The whole was stirred for $5 \mathrm{~h}$ at $55^{\circ} \mathrm{C}$. After the first cycle of $5 \mathrm{~h}$, the mixture was allowed to cool at room temperature. Then, after cooling, another portion of the mixture $($ EP5 + TETA + DODT or TTMA or PETMP) was added to the flask and mixes for next $5 \mathrm{~h}$ at $55^{\circ} \mathrm{C}$. After that, $45 \mathrm{ml}$ of ethanol was imparted to the solution and 
(a)

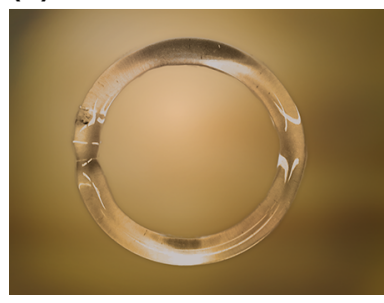

(b)

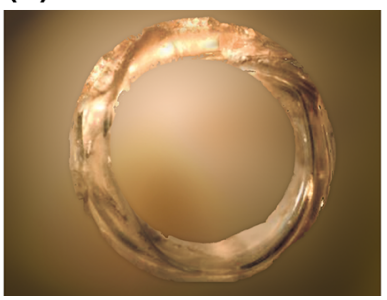

(c)

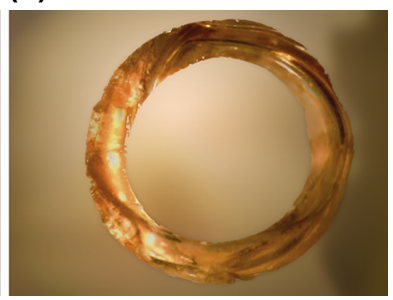

Fig. 1 a Glass ring Fensky and $\mathbf{b}$ glass ring coated with epoxy resin modified by DODT and $\mathbf{c}$ palladium catalysts immobilized on prepared support

Fig. 2 a Chemical structure of the mercaptans 3,6-dioxa-1,8octanedithiol (DODT),

b trimethylopropane tris(3mercaptopropionate) (TTMA), c pentaerythritol tetrakis(3mercaptopropionate) (PETMP) (a)<smiles>SCCOCCOCCS</smiles>

(b)<smiles>CCC(COC(=O)CCS)(COC(=O)CCS)COC(=O)CCS</smiles><smiles>O=C(CCS)OCC(COC(=O)CCS)(COC(=O)CCS)COC(=O)CCS</smiles>

next the mixture was permitted to cool at $24{ }^{\circ} \mathrm{C}$. In the next step, the used solvent was evaporated under vacuum at elevated temperature $80{ }^{\circ} \mathrm{C}$. Prepared carrier was transferred to a Petri dish and dried in an oven at $80{ }^{\circ} \mathrm{C}$ for about $15 \mathrm{~min}$.

\section{Catalyst preparation}

A typical procedure was followed. To a known amount of the support $(0.5 \mathrm{~g})$ in a round-bottom flask, $\mathrm{PdCl}_{2}(\mathrm{PhCN})_{2}$ dissolved in $10 \mathrm{ml}$ of toluene was added. The mixture was stirred at room temperature for 2-3 days when all Pd complex had reacted with the polymer. The arisen amber product was filtrated off, extracted with toluene under nitrogen to remove non-chemically bound to the polymer and also dried under vacuum conditions. For all designed palladium catalysts the metal content was fastened below the metal maximum uptake capacity for an epoxy resin what was also verified by atomic absorption spectroscopy analysis of the colorless toluene solutions after immobilization. The mixture was stirred at room temperature for 3 days, the change of the compound color from dark brown to slightly yellowish indicated that palladium complex had reacted with the prepared polymer. The 


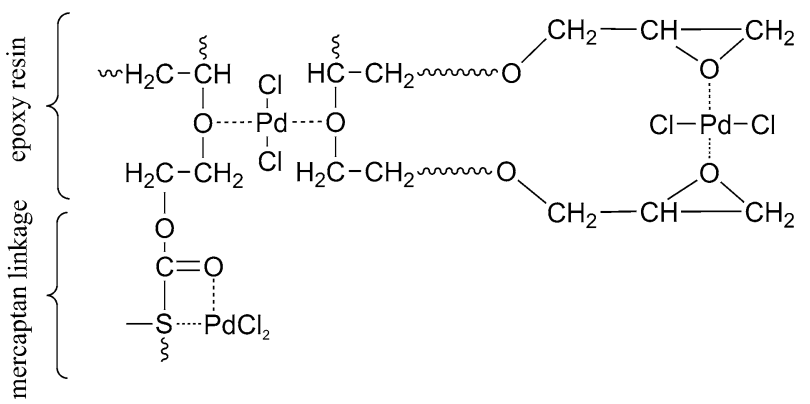

Fig. 3 Possible mode of coordination of Pd(II) complex by mercaptan-modified epoxy support

possible mode of coordination of $\mathrm{Pd}(\mathrm{II})$ complex by mercaptan-modified epoxy support was shown in the Fig. 3.

\section{Catalyst test}

The activity and stability of the palladium catalysts was tested during a multiple use in the model Heck and cinnamaldehyde hydrogenation reaction.

The hydrogenation reactions were carried out in $100 \mathrm{ml}$ Parr microreactor model 4593 with glass liner. The reactor was charged with $0.2 \mathrm{~g}$ of supported palladium catalyst, $5 \mathrm{ml}$ of $96 \%$ ethanol, $0.25 \mathrm{ml}$ of toluene and $0.25 \mathrm{ml}$ of cinnamaldehyde. The reactor was rinsed several times with pure hydrogen and then the hydrogen pressure and temperature were adjusted to the required level, 50 bar and $80{ }^{\circ} \mathrm{C}$, respectively.

The typical Heck reaction was carried out in $50 \mathrm{ml}$ laboratory reactor. The reactor was charged with $0.05 \mathrm{~g}$ of supported palladium catalyst, $0.28 \mathrm{ml}$ $(2.5 \mathrm{mmol})$ of iodobenzene, $0.23 \mathrm{ml}(2.5 \mathrm{mmol})$ of methyl acrylate, $0.15 \mathrm{ml}$ $(1.4 \mathrm{mmol})$ of toluene, $0.35 \mathrm{ml}(2.5 \mathrm{mmol})$ of triethylamine and $1.00 \mathrm{ml}$ (10.4 mmol) of $\mathrm{N}$-methylpyrrolidone (NMP). The reaction was conducted at a temperature of $80^{\circ} \mathrm{C}$ for $1.5 \mathrm{~h}$. Samples were taken every after 5, 15, 30, 60 and $90 \mathrm{~min}$.

The products of both reactions were quantitatively analyzed by gas chromatography (GC) on a Hewlett-Packard 5990 II gas chromatograph equipped with a thermal conductivity detector. The GC column was HP-50 + (cross-linked 50\% Ph Me silicone) $30 \mathrm{~m} \times 0.63 \mathrm{~mm} \times 1.0 \mu \mathrm{m}$ film thickness. The product was identified by matching retention times with those of authentic samples.

In the recycling tests, when reaction was completed, the catalyst was mechanically separated on the sieve, washed with $3 \times 25 \mathrm{ml}$ of toluene, vacuum dried and used for the next reaction.

\section{Mercury test}

The mercury poisoning experiment was performed using 500-fold excess of $\mathrm{Hg}(0)$ relative to palladium catalyst. Polymer-supported catalyst was vigorously stirred 
with mercury for $24 \mathrm{~h}$ prior reaction. The same experiment was also conducted as reference, using homogeneous precatalyst complex as catalyst, $\mathrm{PdCl}_{2}(\mathrm{PhCN})_{2}$. In this case, $\operatorname{Hg}(0)$ was introduced at the beginning, together with other reactants.

\section{Study of Pd leaching}

The amount of palladium loaded in the support was analyzed by atomic absorption spectroscopy (AAS) method with a Perkin Elmer 3030 atomic absorption spectrometer. A sample of a glass polymer-supported catalyst was heated up to $600{ }^{\circ} \mathrm{C}$ and then dissolved in aqua regia. The resulting solution was diluted and assayed by AAS. Time-of-flight secondary ion mass spectrometry (ToF-SIMS) measurements were performed using an ION-TOF GmbH (TOF-SIMS IV) spectrometer with high mass resolution (9000 for $m=29 \mathrm{u}$ ), equipped with the time-of-flight analyzer of reflection type and $\mathrm{Bi}_{3}{ }^{+}$gun working at $25 \mathrm{keV}$. For each sample, three spectra from different surfaces areas were detected. To compare the quantity of palladium present on the surface of "fresh" and "used" catalysts, the number of counts of selected ions obtained from collected mass spectra was normalized on the basis of the value of total counts.

\section{Morphology studies}

The morphology of prepared carriers and in the next stage of made catalysts was defined with a scanning electron microscope JEOL $5500 \mathrm{LV}$, which functioning in high vacuum condition and accelerating voltage of $10 \mathrm{kV}$. Energy-dispersive X-ray microscope (SEM-EDX), JEOL JSM 840A (Japan) was used to observe the elemental distribution. Pore size distribution parameters were determined by application of the BET method on Sorptomatic 1900 FISONS Instrument.

\section{Spectroscopy characterization}

The FT-IR absorption spectra were recorded using BIO-RAD 175C (USA) spectrophotometer in an air atmosphere [16, 17]. X-ray photoelectron spectra (XPS) were prepared on a VG ESCALAB 210 equipment with $\mathrm{Mg} \mathrm{K}$ a $(\mathrm{hm} 51253.6 \mathrm{eV})$ simulation from an X-ray tube (reduced power $10 \mathrm{kV}, 10 \mathrm{~mA}$ ). Before these measurements, ready samples of catalysts and standard sample were pressed to pellets under a pressure of $100 \mathrm{kbar}$ for $10 \mathrm{~min}$. The resolution XPS $S 2 p$, $N 1 s, O 1 s, C l 2 p$, and $\mathrm{Pd} 3 d$ core level recorded spectra were determined. Set the pass energy to $20 \mathrm{eV}$. A take-off angle used in all XPS measurement was the same $\left(90^{\circ}\right)$. Curve fitting had to be carried out using the ECLIPSE data system software. Used system is also needed to describe each of the components of a complex envelope as a Gaussian and Lorentzian sum formula. Shirley background was used to fit the background which is possible applying non-linear model function proportional to the integral of the elastically scattered electrons. All obtained binding energies (BEs) were calibrated to the neutral $C 1 s$ peak at $284.6 \mathrm{eV}$. 


\section{Results and discussion}

\section{Synthesis and characterization of palladium catalysts}

With the use of mercaptans containing - $\mathrm{SH}$ groups to the modification glass-epoxy support (Fig. 2a, b), we received a polymer chain with ligands which chelating the metal complex Fig. 2c). Incorporation of $-\mathrm{SH}$ groups into polymer chains offers a possibility to access new useful matrix. This elaborated procedure carries on to a controlled introduction of bonding groups to the glass-polymeric structure. The metal complex was introduced to the glass-epoxy matrix via ligand exchange reaction and the support microporous structure can affect the selectivity of adopted reactions. The steric requirements of a catalytic centre tied to a matrix may be very different to those of its homogeneous catalysts. The porous structure of the obtained matrix affects the availability of the inside of the swollen polymer so it increases the selectivity by extending diffusion for reaction species. The most interesting is to find out about the porous structure of prepared supports [micropores $(<2 \mathrm{~nm})$ and mesopores $(>2 \mathrm{~nm}, 50<\mathrm{nm})$ ], which are of the same order of magnitude of dimension as those of reacting molecules. Using the common static BET adsorption method, (Table 1) indicated that investigated supports have different pore size distribution which probably arise from differences in their crosslink densities. The catalysts had various pore size distribution with the micropores (radii below $2 \mathrm{~nm}$ ) and very low volume of meso- and macropores. We are aware of the poor accuracy of the BET method for low-porosity materials like highly cross-linked epoxy resin. With regard to the swelling behavior of the resin, especially when the catalytic reaction is performed under solid-liquid heterogeneous conditions, it is important to realize that the dimension of pores can change enormously. Therefore, in future work, we will apply additional techniques such as inverse steric exclusion chromatography (ISEC) and pycnometry to study morphology of these supports and catalysts in the swollen state. In addition for the carrier EP/DODT was observed an insignificant decrease in specific volume of micro-porous after the immobilization of metal (for EP/DODT $72.1 \%$ before immobilization to $71.5 \%$ after

Table 1 Characteristics of glass-polymer matrix and supported palladium catalysts by the BET methods

\begin{tabular}{lllll}
\hline & EP5/PETMP & EP5/PETMP/Pd & EP5/DODT & EP5/DODT/Pd \\
\hline Surface area $\left(\mathrm{m}^{2} \mathrm{~g}^{-1}\right)$ & 89 & 85 & 61 & 59 \\
Average pore diameter $(\mathrm{nm})$ & 245 & 19 & 10 & 5 \\
Volume of pores $(\%)$ & & & & \\
macro $\mathrm{w}>50 \mathrm{~nm}$ & 16,4 & 15.2 & 11.1 & 10.9 \\
meso 2 $<\mathrm{w}<50 \mathrm{~nm}$ & 68,3 & 66.9 & 16.8 & 17.6 \\
micro $\mathrm{w}<2 \mathrm{~nm}$ & 15.3 & 17.9 & 72.1 & 71.5 \\
Pore specific volume $\left(\mathrm{cm}^{3} \mathrm{~g}^{-1}\right)$ & 0.31 & 0.35 & 0.29 & 0.30 \\
Porosity & 0.29 & 0.35 & 0.13 & 0.16 \\
\hline
\end{tabular}



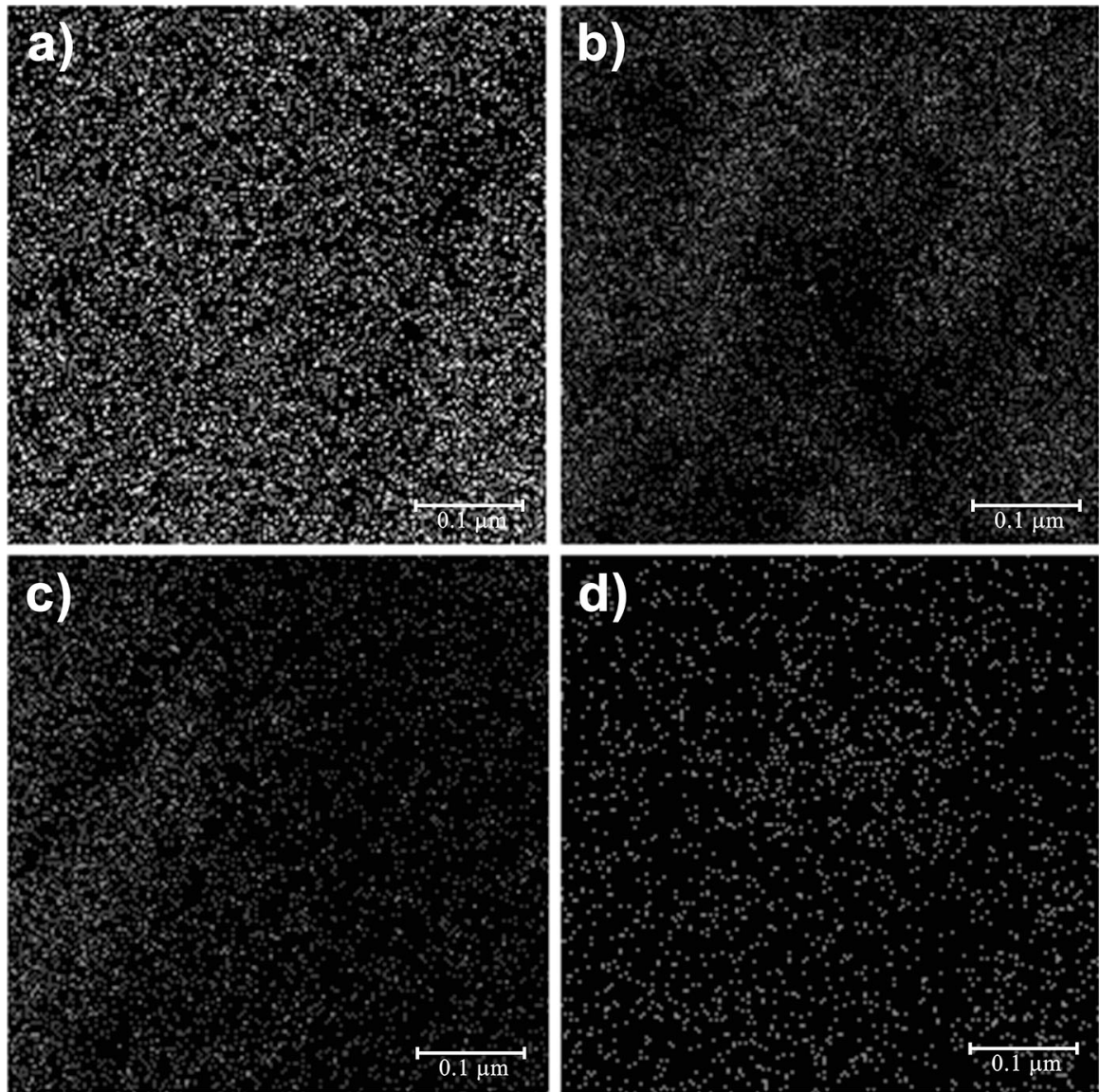

Fig. 4 SEM-EDX micrographs of the surface of the a EP5/DODT/Pd/before hydrogenation reaction, b EP5/DODT/Pd/after hydrogenation reaction, c EP5/TTMA/Pd/before hydrogenation reaction and d EP5/TTMA/Pd/after hydrogenation reaction

immobilization). Such small decrease in the specific volume of micro-porous can indicate a poor blockage of the metal complex in micro-sized pores.

The glass support EP5/PETMP had the inferior value of average pore size, the lower surface area and lower porosity than other supports EP5/DODT and EP5/ TTMA. The available surface area and corresponding decrease in size pore diameter for used supported catalyst was observed.

A scanning electron microscope with energy-dispersive X-ray analyzer (SEMEDX) was used to characterization of palladium distribution on the surface of the matrices and also the distribution of the size of the nanoparticles. The dispersions of the supported palladium catalysts before use in hydrogenation reaction is presented in Fig. 4.

Infrared spectroscopy was used to identify the mode of bonding of the metal species with the functional sites of the polymers. The chemical structure of the 


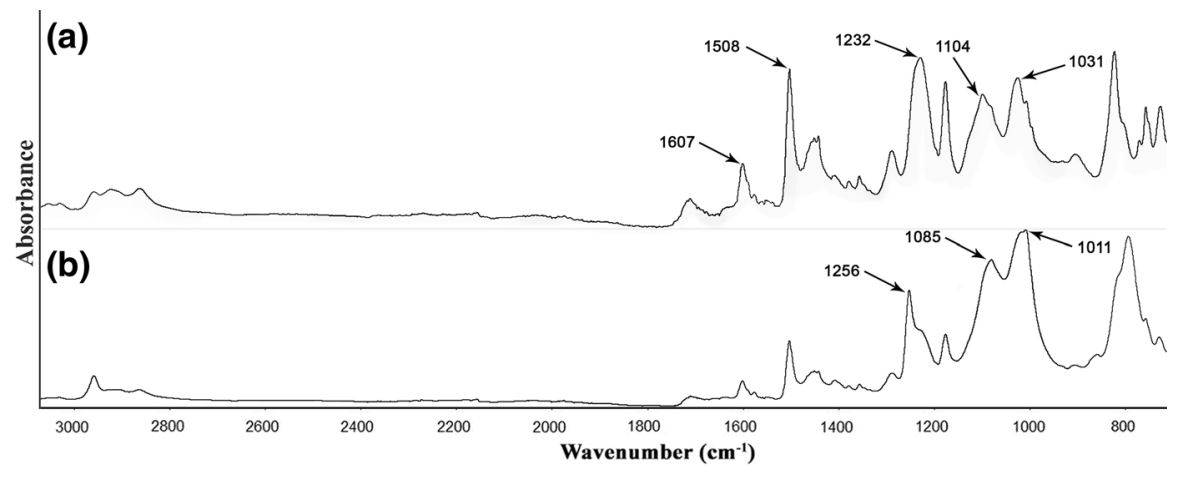

Fig. 5 FT-IR spectra of a EP5/PETMP/Pd; b EP5/PETMP

polymers was confirmed by FT-IR. Figure 5 shows IR spectra of cured epoxy resin with multifunctional mercaptan (PETMP) and prepared catalyst EP5/PETMP/Pd. The lack of absorption peaks of $\mathrm{S}-\mathrm{H}$ vibration at $2545 \mathrm{~cm}^{-1}$ demonstrates the quantitative polyaddition of thiol groups to epoxy system. There is a small peak at $1716 \mathrm{~cm}^{-1}$ and there are no free hydroxyl groups for this catalyst. The shifts and clear changes in the intensity of ether absorption bands at 1011, 1085 and $1232 \mathrm{~cm}^{-1}$ after metal immobilization suggest that ether linkage generated with the opening of each epoxy ring or introduced with mercaptan hardener, is one of the active center of polymer network. The stretching vibration of the $\mathrm{C}=\mathrm{O}$ group of the mercaptan and the presence of characteristic mercaptan bands at $1607 \mathrm{~cm}^{-1}$ gives proof for crosslinking process. The free hydroxyl groups remaining in the condensed resin provide adequate points for epoxy crosslinking via mercaptan linkages. The change in intensity of bands at 1104 and $1508 \mathrm{~cm}^{-1}$ shows the oxygen atoms of mercaptan moiety can participate in metal coordination. The epoxy system modified with mercaptans has a few possible catalytic coordination centers especially here about oxygen and sulfur to which the palladium ion can be bound. It should be noted that although various concentrations of palladium complex were used, the amount of metal attached to epoxide supports remained the same, that is below the sorption capacity of the polymers.

X-ray photoelectron spectroscopy analysis were carried out to study the binding mechanism of $\mathrm{Pd}(\mathrm{II})$ to the glass-polymer matrix. Table 2 shows the electron BEs $(\mathrm{eV})$ of the epoxy resin cured with PETMP as supports before and after

Table 2 Binding energy (eV) values from XPS measurements

\begin{tabular}{llllll}
\hline SAMPLE & O1 s & \multicolumn{2}{l}{$\mathrm{Pd} \mathrm{3d}_{5 / 2}$} & $\mathrm{Cl} 2 \mathrm{p}_{3 / 2}$ \\
\cline { 2 - 5 } & & $\mathrm{Pd}^{\delta+}$ & $\operatorname{Pd}(0)$ & $\operatorname{Pd}(\mathrm{II})$ & \\
\hline EP5/PETMP & 531.62 & - & - & - & - \\
EP5/PETMP/Pd & 534.45 & 336.44 & - & 340.6 & 198.89 \\
before use & & & & & \\
EP5/PETMP/Pd & 534.38 & 335.29 & 335.4 & 340.2 & 198.69 \\
\hline
\end{tabular}


immobilization of Pd complex. EP5/PETMP/Pd catalysts were characterized both prior to and following their use in the Heck reaction. Their XPS spectra exhibited two dominating peaks of $\mathrm{Pd} 3 \mathrm{~d}_{5 / 2}$ and $\mathrm{Pd} 3 \mathrm{~d}_{3 / 2}$ typical for $\mathrm{Pd}(\mathrm{II})$. The XPS data are consistent with FT-IR measurements and show that multiple possible coordination sites assist in binding within the polymer. The increase of the metal nanoparticles becomes limited by the steric restrictions imposed by support nanoscale morphology.

\section{Activity and stability tests}

The structure of tested recyclable glass-polymer supports significantly affect the stability, catalyst activeness and the selectivity-prepared catalysts which was checked in the Heck reaction and hydrogenation of cinnamaldehyde. The selective hydrogenation of $\alpha, \beta$-unsaturated carbonyl compounds to their corresponding unsaturated alcohols is a very important step in the preparation of various fine chemicals such as fragrances for the perfume and food industry. The reaction hydrogenation of $\alpha, \beta$-unsaturated aldehydes which allows to obtain unsaturated alcohols is a rather difficult because the hydrogenation of an alkene double bond catalyzed by noble metals is thermodynamically and kinetically more privileged over the hydrogenation of a carbonyl group. The hydrogenation of cinnamaldehyde generates three primary reaction products, namely hydrocinnamaldehyde (HCALD), cinnamyl alcohol (CALC), and hydrocinnamyl alcohol (HCALC) (Scheme 1).

Table 3 shows the experiment results of the liquid phase hydrogenations of cinnamaldehyde which were carried out under 50 bar pressure at $80{ }^{\circ} \mathrm{C}$ with a stirring rate of $1000 \mathrm{rpm}$. For proved recyclable catalytic systems, immobilized palladium complex $\mathrm{PdCl}_{2}(\mathrm{PhCN})_{2}$ catalysts have been found to give rather weak selectivity to CALC after subsequent use, especially due to the reduction of the

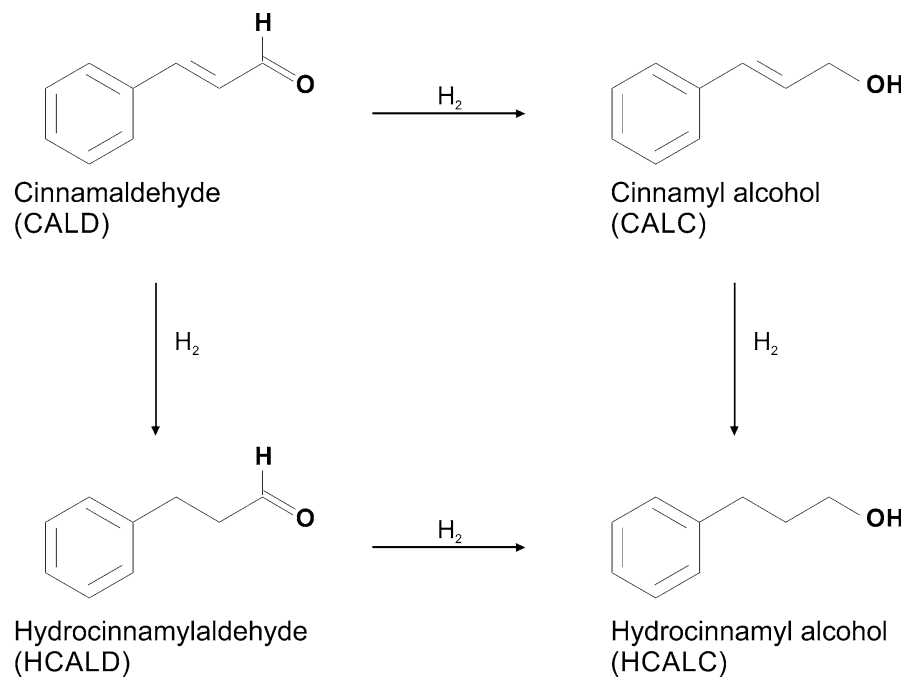

Scheme 1 Hydrogenation of cinnamaldehyde 
Table 3 The reaction hydrogenation of cinnamaldehyde over the EP5/DODT/Pd, EP5/TTMA/Pd and EP5/PETMP/Pd

\begin{tabular}{llllll}
\hline Recycable catalyst & Run & Conversion (\%) & HCALD (\%) & HCALC (\%) & CALC (\%) \\
\hline EP5/DODT/Pd & 1 & 98 & 10 & 67 & 23 \\
& 3 & 96 & 2 & 96 & 2 \\
EP5/TTMA/Pd & 1 & 97 & 8 & 60 & 32 \\
& 3 & 96 & 2 & 97 & 1 \\
EP5/PETMP/Pd & 1 & 97 & 7 & 63 & 30 \\
& 3 & 95 & 3 & 95 & 2 \\
\hline
\end{tabular}

supported precursor. Despite the fact that CALC is strongly adsorbed on the $\operatorname{Pd}(0)$ catalyst surface, it may undergo isomerisation process to the HCALD by which one molecule is transformed into another molecule which has exactly the same atoms, but the atoms have a different arrangement. The catalyst selectivity also appears to depend on the size distribution of the metal nanoparticles generated during the first reaction cycle. The increase of the metal nanoparticles during reduction becomes limited by the steric restrictions superimposed by matrix nanoscale morphology. Dissimilar metal particle size distribution influenced by morphology of the epoxy support can be a clarification for the irregular catalyst performances of the EP5/ PETMP/Pd catalyst, with the smallest size of nanopores. Larger palladium nanoparticles located on EP5/DODT/Pd systems with a larger pore size, were found to give higher selectivity to advisable cinnamyl alcohol. The fairly low selectivities of cinnamyl alcohol were reported for $\mathrm{Ru} / \mathrm{Al}_{2} \mathrm{O}_{3}$ catalyst $(30 \%)$. No cinnamyl alcohol was detected as a neither product of reaction conducted over silica-supported Pd catalysts. In addition, supported iridium, cobalt, osmium, and platinum catalysts have been found to give really weak selectivity to unsaturated alcohols, but high selectivity can be induced by the addition of small amounts of metal chlorides such as $\mathrm{FeCl}_{2}[18,19]$. The activity and selectivity to the target product and stability of the supported catalyst was tested during repeated catalysis runs. Simple and effortless recycling of the immobilized on glass-polymer palladium catalysts showed their potent stability despite the changes which can occur in their structure during the reaction. The assessment of catalytic properties of the developed recyclable catalysts were also analyzed for liquid phase hydrogenation of crotonaldehyde (CAL). The reaction gives the mixture of saturated aldehyde (butyraldehyde-SAL), saturated alcohol (butanol-SOL) and crotyl alcoholUOL. The diacetal (1,1-diethoxybutane-DA) is formed in the reaction between CAL and ethanol used as a solvent. Herein, test results showed in Table 4 that the catalyst selectivity towards crotyl alcohol was changed after each catalytic cycles. The higher selectivity to crotyl alcohol is the result of a large increase in the rate of the hydrogenation of the $\mathrm{C}=\mathrm{O}$ group. A greater selectivity towards $\mathrm{UOL}$ means a low selectivity for the hydrogenation of the $\mathrm{C}=\mathrm{C}$ bond. What is more, a fast conversion of CAL to UOL also means a low yield of the DA.

The features of the palladium catalysts were also researched during their multiple usage in the Heck reaction. The coupling Heck reaction iodobenzene reagent with 
Table 4 Hydrogenation reaction of crotonaldehyde over the EP5/PETMP/Pd and EP5/DODT/Pd catalysts

\begin{tabular}{|c|c|c|c|c|c|c|c|}
\hline \multirow[t]{2}{*}{ Catalyst } & \multicolumn{6}{|c|}{ Distribution of the reaction products } & \multirow{2}{*}{$\begin{array}{l}\text { DA } \\
(\%)\end{array}$} \\
\hline & Run & $\begin{array}{l}\text { Conversion } \\
(\%)\end{array}$ & $\begin{array}{l}\text { CAL } \\
(\%)\end{array}$ & $\begin{array}{l}\text { SAL } \\
(\%)\end{array}$ & $\begin{array}{l}\text { SOL } \\
(\%)\end{array}$ & $\begin{array}{l}\text { UOL } \\
(\%)\end{array}$ & \\
\hline $\begin{array}{l}\text { Homogeneous } \\
\mathrm{PdCl}_{2}(\mathrm{PhCN})_{2}\end{array}$ & 1 & 98 & 14 & 86 & - & - & - \\
\hline \multirow[t]{2}{*}{ EP5/PETMP/Pd } & 1 & 97 & 24 & 10 & 13 & 29 & 24 \\
\hline & 5 & 96 & 3 & 12 & 31 & 34 & 20 \\
\hline \multirow[t]{2}{*}{ EP5/DODT/Pd } & 1 & 98 & 24 & 9 & 10 & 28 & 29 \\
\hline & 5 & 97 & 5 & 14 & 29 & 37 & 15 \\
\hline
\end{tabular}

Conditions: Temperature $90{ }^{\circ} \mathrm{C}$, time $8 \mathrm{~h}$; solvent: $96 \%$ ethanol; the overall volume: $5.5 \mathrm{~mL}$; unidentified reaction products are not shown

methyl acrylate reagent, occurring in the solution of $N$-methylpyrrolidone (NMP) in the presence of triethylamine. The only observed product of the reaction was methyl trans-cinnamate (Scheme 2).

The catalytic activity of all formulated systems was similar to homogeneous palladium precursor $\mathrm{PdCl}_{2}(\mathrm{PhCN})_{2}$ (Table 5). The slighter activity of the palladium catalysts supported on the EP5/PETMP we assign to fewer Pd sites being approachable to the reactants when these are attached to the polymer of minor microporosity. The easier availability of the catalytic sites situated in a DODT-and

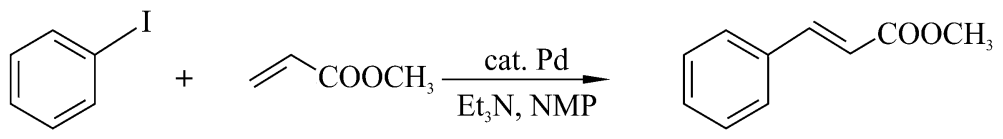

Scheme 2 Heck reaction

Table 5 The catalyst activity in Heck reaction

\begin{tabular}{lllll}
\hline Catalyst & Run & $\begin{array}{l}{ }^{106} \mathrm{Pd}^{+} \text {intensity of } \\
\text { emission }^{\mathrm{a}}\end{array}$ & $\begin{array}{l}\text { Conversion } \\
(\%)^{\mathrm{b}}\end{array}$ & $\begin{array}{l}\mathrm{Pd}^{\mathrm{c}} \text { content }(\mathrm{mmol} / \\
\mathrm{g})^{\mathrm{c}}\end{array}$ \\
\hline $\begin{array}{l}\text { Homogeneous } \\
\mathrm{PdCl}_{2}(\mathrm{PhCN})_{2}\end{array}$ & 1 & - & 98 & - \\
EP5/PETMP/Pd & 1 & $7.5 \times 10^{-3}$ & 97 & 0.0018 \\
& 5 & $5.1 \times 10^{-3}$ & 94 & 0.0014 \\
EP5/DODT/Pd & 1 & $4.8 \times 10^{-3}$ & 89 & 0.0019 \\
& 5 & $3.4 \times 10^{-3}$ & 78 & 0.0012
\end{tabular}

${ }^{a}$ Normalized intensity of ${ }^{106} \mathrm{Pd}^{+}$selected from the mass spectra of palladium catalysts (ToF-SIMS)

${ }^{\mathrm{b}}$ Conversion of iodobenzene after $1.5 \mathrm{~h}$. Reaction conditions: temperature $80{ }^{\circ} \mathrm{C}$; substrates: iodobenzene $0.28 \mathrm{~mL}(2.5 \mathrm{mmol})$, methyl acrylate $0.23 \mathrm{~mL}(2.5 \mathrm{mmol})$; solvent: toluene $0.15 \mathrm{~mL}(1.4 \mathrm{mmol})$, triethylamine $0.35 \mathrm{~mL}$ ( $2.5 \mathrm{mmol}), \mathrm{N}$-methylpyrrolidone (NMP) $1.00 \mathrm{~mL}$ (10.4 mmol); supported catalyst: $0.05 \mathrm{~g}$;. The only observed product was the methyl trans-cinnamate

${ }^{c}$ The amount of palladium loaded in the support was determined by AAS method 
TTMA-modified epoxy resins characterizing a greater volume of micropores and major surface area, increases the activity of supported Pd systems. The activities of all prepared and tested catalyst systems decrease with the subsequent usage which might be most likely as a result of the palladium leaching. ToF-SIMS method allows the estimation of the chemical composition of the catalyst surface and the deactivation process of catalysts, the nature of interactions between metallic phases and supports as well as the distribution of metal or other catalyst components on the surface. ToF-SIMS spectrometry may be very useful in the studies of catalyst deactivation processes but unfortunately the quantitative analysis by TOF-SIMS technique is relatively difficult. Heterogenized catalysts after repeated use (5th use) in Heck reaction were analyzed and compared to the fresh samples. A really small loss of the amount of surface-approachable palladium was observed; however, the drop of Pd content was at a safe level and did not significantly influence the activity.

\section{Conclusion}

The developed and prepared glass-polymer matrices made with glass core and successfully used mercaptan-modified epoxy resin as shell in encapsulation process, meet the given requirements for catalyst supports in that they may be simply synthesized in a form suitable for heterogenization reaction of palladium complex catalysts and do not need to work efficiently any additional functionalization. The biggest advantage, apart from the catalytic properties of the prepared systems there is that this novel type of supports for palladium complex catalysts may be in easy way mechanical separated from the conducted reaction. Results obtained during the tests have proved that the morphology of the polymer carriers in the model catalytic reaction (Heck reaction and hydrogenation reaction) occurs to have more effect on the reaction characteristic, activity and selectivity as compared with the impact of the chemical structure of the matrices. The type and amount of mercaptan used as resin modifier can have a significant impact on the morphology aspects such as pore size distribution and particular surface area prepared systems. The enhancement of the catalytic activity of supported Pd catalysts is caused by the better availability of the catalytic sites situated in modified resins having a greater volume of microspores and larger superficies area. All severe and recyclable glass-polymer palladium catalysts have had a potent catalytic stability for consecutive runs both before and after recycling efficiency tests. In addition, the next research will focus on the application of these systems for other organic reactions and characterization of the mode of bonding between the palladium atoms and the functional groups of the mercaptan-modified epoxy resin.

Open Access This article is distributed under the terms of the Creative Commons Attribution 4.0 International License (http://creativecommons.org/licenses/by/4.0/), which permits unrestricted use, distribution, and reproduction in any medium, provided you give appropriate credit to the original author(s) and the source, provide a link to the Creative Commons license, and indicate if changes were made. 


\section{References}

1. Suriboot J, Hobbs CE, Yang Y, Bergbreiter DE (2012) Protective encapsulation of acid-sensitive catalysts using polyethylene ligands. J Polym Sci 50:4840-4846. https://doi.org/10.1002/pola.26319

2. Mennecke K, Cecilia R, Glasnov TN, Gruhl S, Vogt C, Feldhoff A, Vargas L, Kappe O, Kunz U, Kirschninga A (2008) Palladium(0) nanoparticles on glass-polymer composite materials as recyclable catalysts: a comparison study on their use in batch and continuous flow processes. Adv Synth Catal 350:717-730. https://doi.org/10.1002/adsc.200700510

3. Kirschning A, Solodenko W, Mennecke K (2006) Combining enabling techniques in organic synthesis: continuous flow processes with heterogenized catalysts. Chem Eur J 12:5972-5990. https:// doi.org/10.1002/chem.200600236

4. Jacinto MJ, Silva FP, Kiyohara PK, Landers R, Rossi LM (2012) Catalyst recovery and recycling facilitated by magnetic separation: iridium and other metal nanoparticles. ChemCatChem 4:698-703. https://doi.org/10.1002/cctc.201100415

5. Bączek N, Strzelece K, Wąsikowska K (2013) Magnetic recykling of complex catalysts immobilized on thiol-functionalized polymer supports. Pol J Chem Technol 15(3):65-68. https://doi.org/10.2478/ pjct-2013-0046

6. Veisia H, Gholami J, Ueda H, Mohammadia P, Noroozi M (2015) Magnetically palladium catalyst stabilized bydiaminoglyoxime-functionalized magnetic $\mathrm{Fe}_{3} \mathrm{O}_{4}$ nanoparticles as active and reusable catalyst for Suzuki coupling reactions. J Mol Catal A Chem 396:216-223. https://doi.org/10.1016/j. molcata.2014.10.012

7. Mei N, Liu B, Zheng J, Lv K, Tang D, Zhang Z (2015) A novel magnetic palladium catalyst for the mild aerobic oxidation of 5-hydroxymethylfurfural into 2,5-furandicarboxylic acid in water. Catal Sci Technol 5:3194. https://doi.org/10.1039/c4cy01407c

8. Sienkiewicz N, Strzelec K, Pospiech P, Cypryk M, Szmechtyk T (2016) New palladium catalyst immobilized on epoxy resin: synthesis, characterization and catalytic activity. Appl Organomet Chem 30:4-11. https://doi.org/10.1002/aoc.3389

9. Kunz U, Schonfeld H, Kirschning A, Solodenko W (2003) Polymer/carrier composites as materials and reactors for organic synthesis. J Chromatogr A 1006:241-249. https://doi.org/10.1016/S00219673(03)00556-9

10. Mannar M (2012) Catalytic applications of polymer-supported molybdenum complexes in organic transformations. Curr Org Chem 16(16):73-88. https://doi.org/10.2174/138527212798993130

11. Ostrowska S, Markiewicz B, Wąsikowska K, Bączek N, Pernak J, Strzelec K (2012) Epoxy resins cured with ionic liquids as novel supports for metal complex catalysts. Cr Chim 16:752-760. https:// doi.org/10.1016/j.crci.2012.12.005

12. Dergunov S, Khabiyev A, Shmakov S, Kim M, Ehterami N, Weiss M, Birman V, Pinkhassik E (2016) Encapsulation of Homogeneous catalysts in porous polymer nanocapsules produces fastacting selective nanoreactors. ACS Nano 10(12):11397-11406. https://doi.org/10.1021/acsnano. $6 \mathrm{~b} 06735$

13. Ye R, Zhukhovitskiy A, Deraedt C, Toste F, Somorjai G (2017) Supported dendrimer-encapsulated metal clusters: toward heterogenizing homogeneous catalysts. Acc Chem Res 50(8):1894-1901. https://doi.org/10.1021/acs.accounts. $7 b 00232$

14. Tadepalli S, Yim J, Madireddi K, Luan J, Naik R, Singamaneni S (2017) Gold nanorod-mediated photothermal enhancement of the biocatalytic activity of a polymer-encapsulated enzyme. Chem Mater 29(15):6308-6314. https://doi.org/10.1021/acs.chemmater.7b01527

15. Gross E, Toste F, Somorjai G (2015) Polymer-encapsulated metallic nanoparticles as a bridge between homogeneous and heterogeneous catalysis. Catal Lett 145:126-138. https://doi.org/10.1007/ s10562-014-1436-9

16. Strzelec K, Baczek N, Ostrowska S, Wąsikowska K, Szynkowska MI, Grams J (2012) Synthesis and characterization of novel polythiourethane hardeners for epoxy resins. Cr Chim 15:1065-1071. https://doi.org/10.1016/j.crci.2012.09.003

17. Strzelec K, Baczek N, Szynkowska M, Grams J (2014) Thiirane resins cured with polythiourethane hardeners as novel supports for metal complex catalysts. J Appl Polym Sci 131:1-9. https://doi.org/ 10.1002/APP.40330 
18. Cairns GR, Cross RJ, Stirling D (1997) Hydrogenation of cinnamaldehyde using catalysts prepared from supported palladium phosphine complexes. J Catal 166(9):89-97. https://doi.org/10.1006/jcat. 1997.1491

19. Chambers A, Jackson SD, Stirling D, Webb G (1997) Selective hydrogenation of cinnamaldehyde over supported copper catalysts. J Catal 168:301-314. https://doi.org/10.1006/jcat.1997.1683 